\title{
Design of an Anthropomorphic Respiratory Phantom for PET Imaging
}

\author{
David G. Black ${ }^{1}$, Yas Oloumi Yazdi ${ }^{1}$, Jeremy Wong ${ }^{1}$, Roberto \\ Fedrigo $^{1,2}$, Carlos Uribe ${ }^{3,4}$, Dan J. Kadrmas ${ }^{5}$, Arman Rahmim ${ }^{1,2,4, *}$, \\ Ivan S. Klyuzhin ${ }^{2,4}$ \\ ${ }^{1}$ Department of Physics and Astronomy, University of British Columbia, Vancouver, Canada \\ ${ }^{2}$ BC Cancer Research Institute, Vancouver, Canada \\ 3 Department of Functional Imaging, BC Cancer, Vancouver, Canada \\ ${ }^{4}$ Department of Radiology, University of British Columbia, Vancouver, Canada \\ ${ }^{5}$ Department of Radiology and Imaging Sciences, University of Utah, Salt Lake City, UT, \\ United States \\ * Corresponding Author, Email: arman.rahmim@ubc.ca
}

\begin{abstract}
Purpose: Respiratory motion during positron emission tomography (PET) can be a major detriment to image quality in oncological imaging, causing loss of quantification accuracy and false negative findings. The impact of motion on lesion quantification and detectability can be assessed using anthropomorphic phantoms with realistic anatomy representation and motion modeling. In this work we design and build such a phantom, with careful consideration of system requirements and detailed force analysis.

Methods: We start from a previously-developed anatomically accurate shell of a human torso and add elastic lungs with a highly controllable actuation mechanism which replicates the physics of breathing. The space outside the lungs is filled with a radioactive water solution. To maintain anatomical accuracy in the torso and realistic gamma ray attenuation, all motion mechanisms and actuators are positioned outside of the phantom compartment. The actuation mechanism can produce a plethora of custom respiratory waveforms with breathing rates up to 25 breaths per minute and tidal volumes up to $1200 \mathrm{~mL}$.

Results: Several tests were performed to validate the performance of the phantom assembly, in which the phantom was filled with water and given respiratory waveforms to execute. All parts demonstrated nominal performance. Force requirements were not exceeded and no leaks were detected, although continued use of the phantom is required to evaluate wear. The respiratory motion was determined to be within a reasonable realistic range.

Conclusions: The full mechanical design is described in this paper, as well as a software application with graphical user interface which was developed to plan and visualize respiratory patterns. Both are available open source and linked in this paper. The developed phantom will facilitate future work in evaluating the impact of respiratory motion on lesion quantification and detectability.
\end{abstract}

Keywords: PET, Quantitative Imaging/Analysis, Motion Management, Phantoms - Physical, Motion Correction, Anthropomorphic Phantom 


\section{Contents}

$\begin{array}{lr}\text { I. Introduction } & 1\end{array}$

II. Materials and Methods $\quad 3$

II.A. Lungs . . . . . . . . . . . . . . . . . . . . . . 6

II.B. Breathing Mechanism . . . . . . . . . . . . . . . . . . 8

II.B.1. Piston and cylinder . . . . . . . . . . . . . . . . 8

II.B.2. Mounting Plates . . . . . . . . . . . . . . . . . 11

II.B.3. Linear Actuator . . . . . . . . . . . . . . . . . . . . . . . 12

II.C. Software and Electrical Design . . . . . . . . . . . . . . . . . . 15

II.C.1. USB Interface . . . . . . . . . . . . . . . . . . . . . . 15

II.C.2. Analog Position Control .................. 17

III. Results: Testing and Validation $\quad 18$

$\begin{array}{ll}\text { IV. Discussion } & 20\end{array}$

V. Conclusion $\quad 21$

$\begin{array}{ll}\text { VI. Open Source } & 22\end{array}$

$\begin{array}{lr}\text { VII.Acknowledgements } & 22\end{array}$

$\begin{array}{ll}\text { References } & 22\end{array}$ 


\section{Introduction}

Positron emission tomography (PET) imaging is widely used in oncology for tumor detection, monitoring, and quantification. A leading factor that can adversely affect the quality of thoracic PET images is respiratory motion during the scans ${ }^{1}$. One of the main negative effects is the reduction of contrast for small lesions (e.g. cancer metastases) ${ }^{2}$, which worsens lesion detectability and introduces bias in the measured standardized uptake values (SUVs). For larger lesions, respiratory motion may introduce bias in radiomic features that quantify their shape and texture ${ }^{345}$. In normal respiration, the amplitude of diaphragm movement is on the order of $1.5-2 \mathrm{~cm}^{6}$, while deep inspiration may result in $7-13 \mathrm{~cm}$ diaphragm displacement $^{7}$. For clinical PET scanners with spatial resolution around $0.4-0.5 \mathrm{~cm}$ full-width at half-maximum (FWHM), motion of this magnitude can lead to effective usable resolution closer to $1 \mathrm{~cm}^{89}$. Thus, motion needs to be taken into account in the process of cancer diagnosis and staging ${ }^{10}$. as well as during image-guided radiotherapy ${ }^{11}$.

Since it is impossible to know the ground truth with real subjects, the impact of motion on PET image quantification is typically assessed using geometrically simple phantoms that simulate motion with a precisely-known trajectory, such as the QUASAR Phantom (Modus QA, Ontario, Canada). On the other hand, with continuous advances in PET scanner sensitivity and resolution ${ }^{12}$, as well as development of novel image quantification paradigms ${ }^{13}$, it becomes of interest to evaluate the effects of respiratory motion under more anatomically realistic conditions. Several anthropomorphic phantoms have been developed in the past, each of which solves a subset of the problems associated with modeling respiratory motion. These phantoms are compared in Table 1 . While Wilhelm ${ }^{14}$ has the most realistic overall motion of any existing device, it lacks any bone structure, which greatly affects the realism of the phantom as gamma ray attenuation and scatter by bones can significantly affect the image quality and quantification. Conversely, Alderson ${ }^{15}$ has bones as well as respiratory and cardiac motion; however its anatomy is simplified. It also does not support the addition of lesions, which are key in any studies investigating the effect of motion on image quantification.

The goal of this work was to create an anatomically accurate phantom with realistic modeling of respiratory motion. We build upon an existing phantom previously developed and tested by Kadrmas et al. ${ }^{1617}$, henceforth referred to as "Probe-IQ" (Image Quality Probe). To the best of our knowledge, Probe-IQ to this day has the highest level of anatom- 
ical realism in the field. While highly realistic in PET and, to a lesser extent, computed tomography (CT) scans, the phantom has previously been completely static and not used to study motion effects. The motion-enabled phantom described in this paper uses the shell and rib-cage of the original Probe-IQ phantom, but adds custom-built elastic lungs with trachea, and an electronically-controlled actuation mechanism to create realistic respiratory motion. The lungs expand and contract during the respiration.

The Probe-IQ phantom enhanced with respiratory motion aims to achieve the following: Anatomical Accuracy

- Builds off the static anatomical accuracy of Probe-IQ and has realistic lung motion.

- Avoids any unrealistic parts like connecting rods or metal components in the torso.

- All materials carefully selected with reference to mass attenuation coefficients ${ }^{18}$ that resemble human tissue.

\section{Breathing Control}

- Able to achieve a wide range of breathing rates and tidal volumes (i.e. air displaced between inhalation and exhalation). Namely, at least up to 25 breaths per minute at $1 \mathrm{~L}$ tidal volume; typical relaxed breathing is $500 \mathrm{~mL}$ at $15-20$ breaths per minute ${ }^{19}$.

- Has highly controllable, repeatable, consistent breathing motion to generate high quality data, but also can include occasional disrupted breaths or inconsistencies, as would happen in a real patient.

In addition, safety is a concern, as the phantom is filled with about $15 \mathrm{~L}$ of radioactive solution (300 to $1000 \mathrm{MBq}$ of radioactivity per scan) for PET acquisitions. The design must avoid leaks, and minimize the possibility of large spills. Easy fill-ability of the phantom is also desirable to reduce radiation exposure to users and to increase usability.

Table 1: Comparison of existing anthropomorphic phantoms. CM: cardiac motion, RM: respiratory motion, LM: liver motion, SS: skeletal structure.

\begin{tabular}{l|l|l|l|l|l|l} 
Name & PET/CT/MRI & CM & RM & LM & SS & Lesions \\
\hline Probe-IQ + Respiratory & $\checkmark / \checkmark / \times$ & $\times$ & $\checkmark$ & $\checkmark$ & $\checkmark$ & $\checkmark$ \\
motion capabilities & & & & & & \\
Probe-IQ $^{1617}$ & $\checkmark / \checkmark / \times$ & $\times$ & $\times$ & $\times$ & $\checkmark$ & $\checkmark$ \\
Wilhelm $^{14}$ & $\checkmark / \checkmark / \checkmark$ & $\checkmark$ & $\checkmark$ & $\checkmark$ & $\times$ & $\checkmark$ \\
Alderson $^{15}$ & $\checkmark / \checkmark / \times$ & $\checkmark$ & $\checkmark$ & $\times$ & $\checkmark$ & $\times$
\end{tabular}




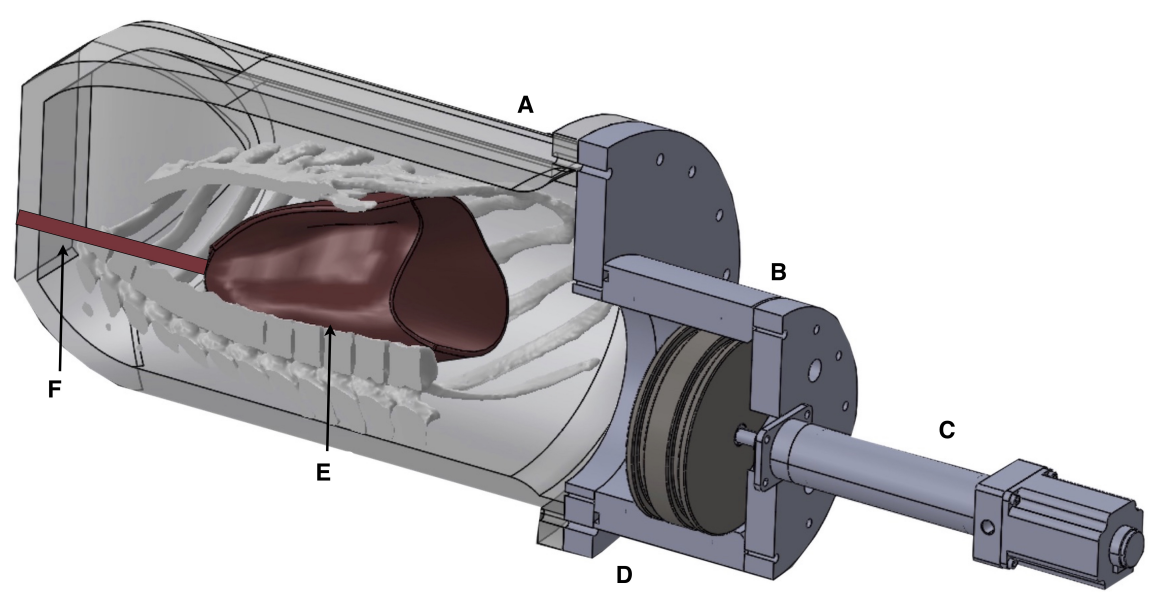

Figure 1: Overview of phantom design. A: existing thorax shell and bone structure (liver and heart compartments included but not pictured). B: Piston and cylinder for torso volume modulation. C: linear actuator. D: custom-designed base-plate allows compatibility with existing Probe-IQ phantom. E: flexible lung inserts of silicone elastomer. F: breathing tubes (trachea) connect lungs to atmosphere to allow breathing.

\section{Materials and Methods}

The respiratory motion-enabled Probe-IQ phantom involves three main components: 1) elastic lungs, 2) an actuation method, and 3) a control system (electronics and software). The overall structure can be seen in Fig. 1 and all design files and code can be accessed in the Open Source Section at the end of this paper. The lungs are located inside the rib cage of the existing Probe-IQ phantom (A), and surrounded by a radioactive water solution creating the warm, non-specific background typically present in oncologic PET images. The lungs are connected to the atmosphere by a breathing tube (trachea) (F), which can be sealed at the neck. The actuating mechanism is positioned below the phantom $(\mathrm{B}, \mathrm{C}, \mathrm{D})$, where the pelvis of a human would be located. It is outside the field of view of the PET scanner so as not to create image artefacts. The phantom operates by piston-actuated passive breathing, as explained below and seen in figure 2 .

Achieving respiratory motion with the lungs is possible through a variety of mechanisms, which fall into two main classes.

First, the lungs can be directly inflated using air pumps or compressors. This is the most obvious approach, but involves a number of complicating factors. The lung volume cannot simply increase inside a sealed, rigid torso filled with an incompressible fluid. An overflow tube or expanding section of the torso would be required. The former would create the risk of leakage, while the latter would require a powerful compressor. Furthermore, 


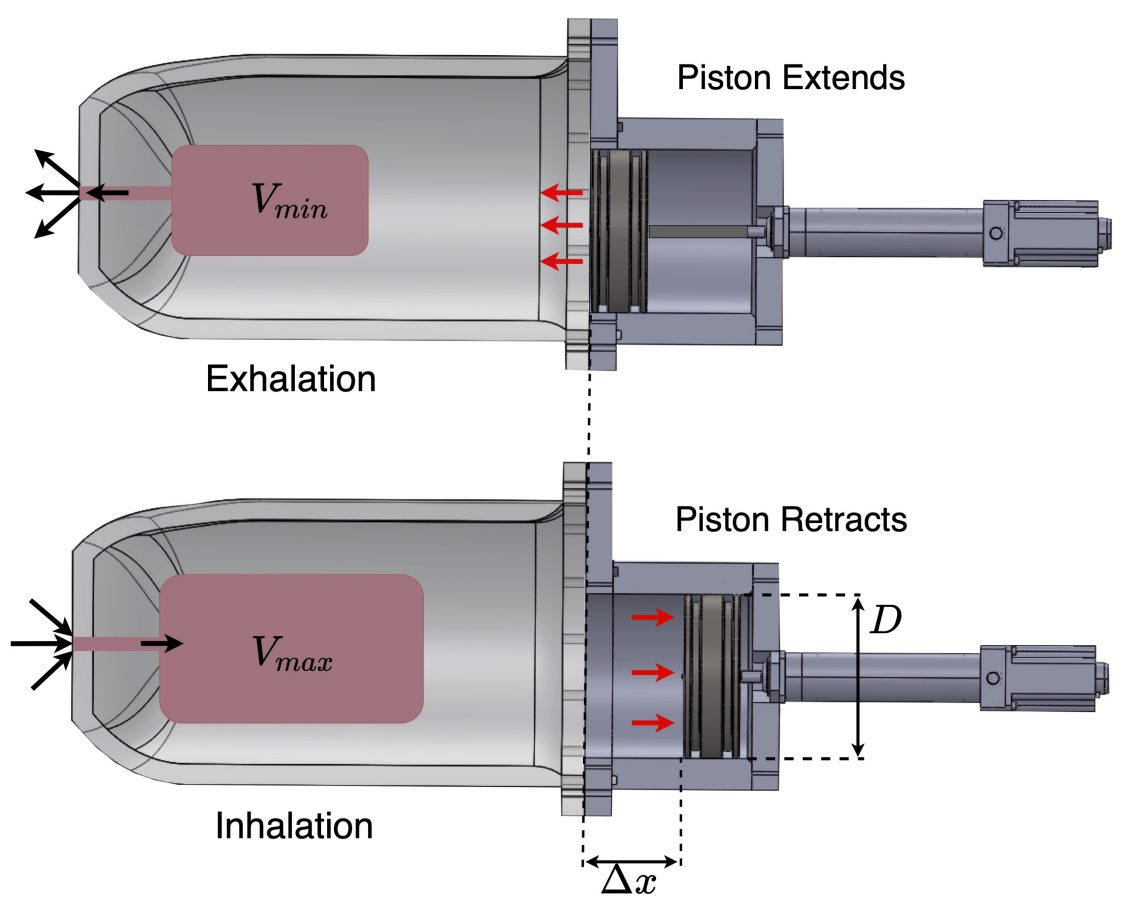

Figure 2: Respiration modeling by modulating the volume of the phantom, mediated by incompressible fluid. Air flows into and out of the lungs through the trachea as the piston extends and retracts.

control is much more difficult with a pneumatic system and would require a great increase in complexity.

The second, and preferred method is to instead modulate the volume or pressure of the whole phantom in a manner analogous to actual respiratory breathing. Since the phantom is sealed, rigid, and entirely filled with water, except for the air-filled lungs, changing its volume by $\Delta V$ induces an equal volume change of $\Delta V$ in the lungs. Instead of compressing the air in a sealed set of lungs, the pressure requirement of the actuating mechanism can be dramatically decreased by opening the lungs to the atmosphere once the phantom has been filled and sealed. Contrary to intuition, the lungs will not collapse under the pressure of the water since the water volume is constant and cannot expand to fill the vacant space created if the lungs were to collapse. In effect, a relative negative pressure is created in the liquid anterior to the lungs, just as human breathing relies on negative intrapleural pressure. Now, when the volume of the torso is changed, that volume of air is simply exhaled to or inhaled from the atmosphere (figure 2). 


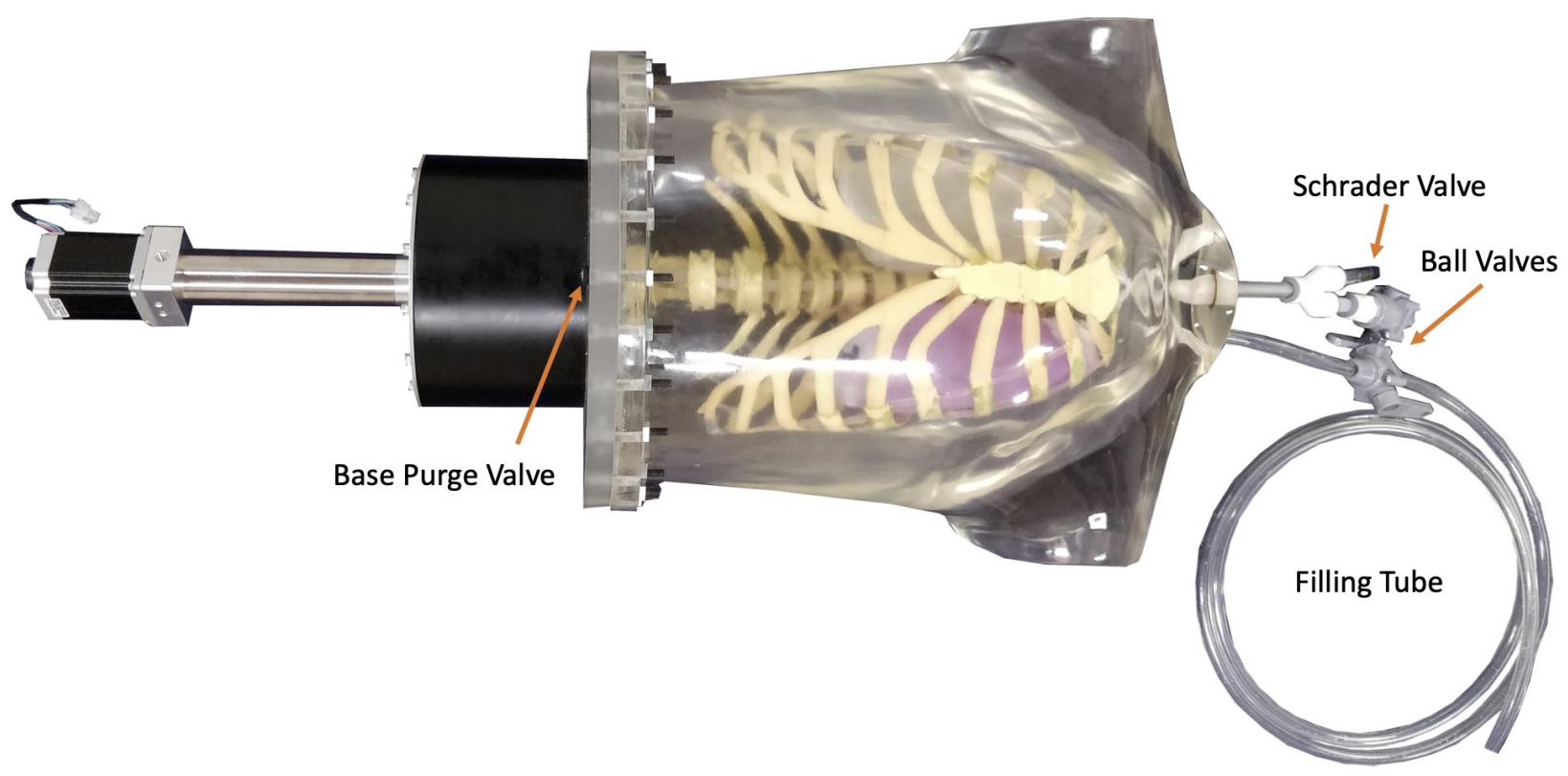

Figure 3: Assembled phantom with filling system

To prepare the phantom for imaging, the following steps are taken:

1. The lungs, organs, and bones are positioned inside the thorax.

2. The lungs are inflated to their maximum capacity via a Schrader valve and a manual pump and sealed (figure 3).

3. The piston is moved to its most retracted position (i.e. the phantom is at maximum volume), and the base plate is attached.

4. The torso is completely filled with radioactive water solution using the ports in the neck, without leaving air pockets. A valve in the base plate, at the highest point in the torso, is used to release the displaced air (figure 3).

5. The torso is sealed, and the lungs opened to the atmosphere.

Since the torso is rigid and sealed, its volume cannot change, so the lungs remain inflated. At this point, the lung volume can be fine-tuned by opening the water-filling port and/or base purge valve while pumping air into the lungs through the Schrader valve using a manual pump. Thus, any small changes or issues can be rectified. With the phantom set up, the user can define a wide variety of respiratory patterns, rates, and volumes using a graphical user interface. The piston then moves along the defined trajectory for the duration of a scan, causing the lungs to expand and contract due to the changes in pressure. The piston motion 
can also be coupled directly to the lungs using thin cables, which are not visible in the PET scan. The next three sections explain the lungs, breathing mechanism, and software in more detail.

\section{II.A. Lungs}

The lung shapes were derived from a CT scan of an adult male lung and simplified using mesh editing software (Blender Foundation, Amsterdam) to reduce the cardiac notch and make the right and left lungs more symmetrical (figure 4). This was done primarily for ease of fabrication, but also to avoid unpredictable expansion and contraction of complex shapes which differ between the lungs. The lungs were designed to have a realistic adult size postexhalation, with a volume of $1650 \mathrm{ml}$ each. This ensures that during normal breathing the lungs only expand, and do not compress or fold in an irregular manner. The dimensions of the lungs are approximately $18.0 \mathrm{~cm}$ (height) by $10.0 \mathrm{~cm}$ (width) by $12.7 \mathrm{~cm}$ (depth) (figure 4 ). The lungs have uniform wall thickness of $2 \mathrm{~mm}$ and can optionally be coupled directly to the piston using thin cables to force a specific longitudinal flex pattern.

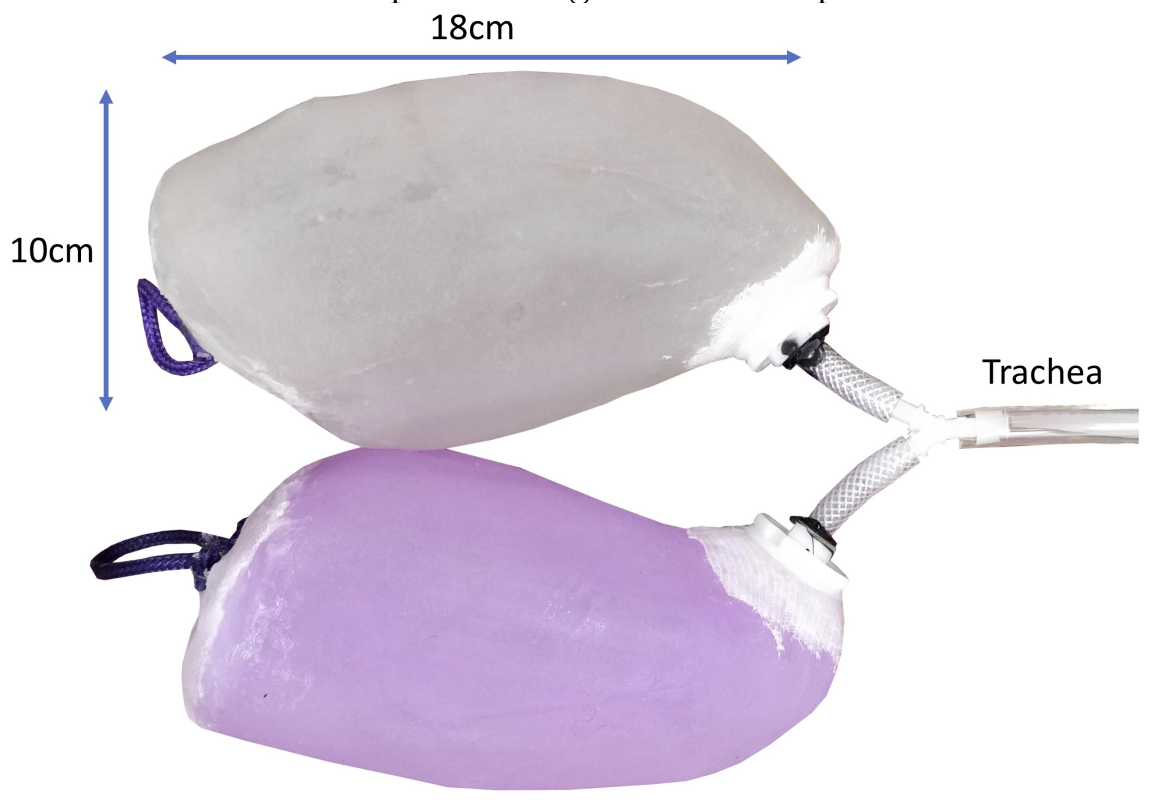

Figure 4: Elastic silicone lungs with bronchi and trachea. Bottom loops can be used to couple the lung motion directly to the piston. Both lungs are the same material with different dyes, used to differentiate between the left and right lung.

A thru-wall fitting commonly used in prosthetics was custom manufactured using Delrin plastic (figure 5). This is attached medially near the top points of both lungs to connect air tubes which lead through a Y-connector to a single tube to the outside of the phantom, 
much like bronchi connecting to the trachea. One of three ports in the neck area of the phantom provides the inlet for the breathing tubes. The lungs are be held in place in the superior/inferior axis using the trachea, and in the lateral and anterior/posterior axes using the rib cage. At the inlet of the trachea, near the neck of the phantom, another Y-connector splits the airway into a large ball valve and a Schrader valve which are used to alternatively pressurize and seal the lungs or allow free airflow (figure 3).
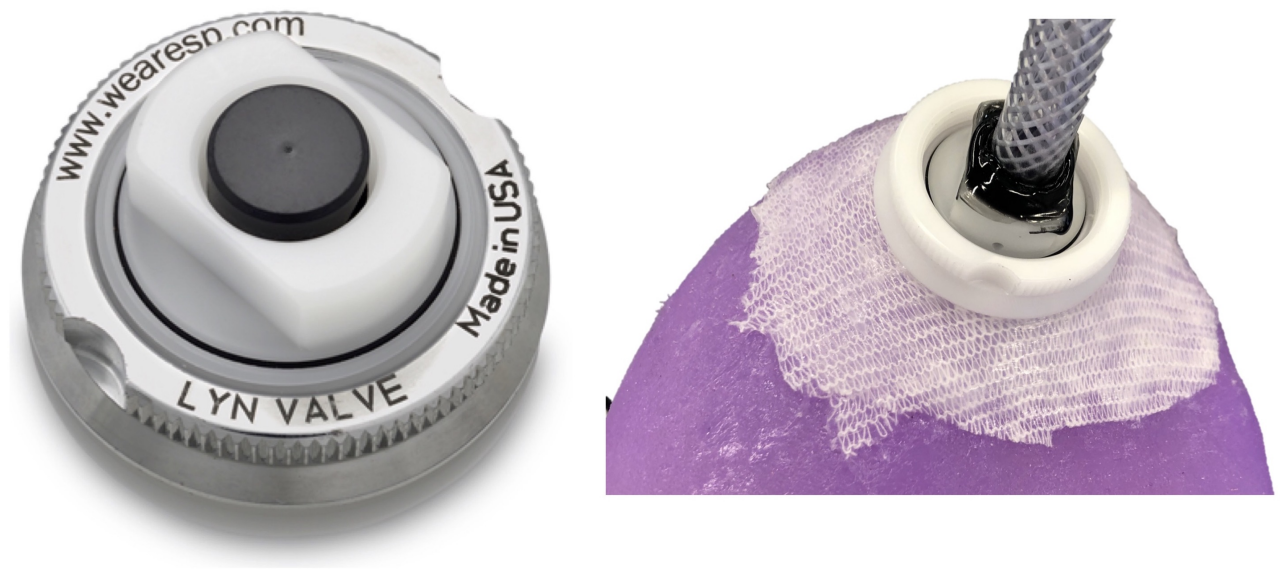

Figure 5: Original (left) and custom manufactured plastic (right) thru-wall fitting (ESP LLC, New Jersey, USA; Image from ESP) used to attach trachea to the lungs. The lung silicone is compressed between the twin flanges and creates an air-tight seal.

The lungs were manufactured from Chlorosil-35 (Ottobock, Germany), a two-part, silicone-based elastomer with Shore A hardness 35. Following high temperature vulcanization, this material is durable, long-lasting, and thermostable, yet flexible to allow contraction and expansion. The material was previously tested in another phantom ${ }^{14}$. The lung manufacturing process was as follows:

1. A negative mold was 3D-printed out of polylactic acid (PLA) in two halves due to size constraints in 3D-printing.

2. Dental plaster was poured into the negative mold using a hole at the base.

3. Once the plaster dried, it was extracted from the mold and put in a polyvinyl alcohol (PVA) bag. This bag prevents the Chlorosil catalyst from reacting with the plaster.

4. The Chlorosil was rolled onto the positive dental plaster mold of the lungs and placed in an evacuated plastic bag to obtain a uniform shape and thickness.

5. The mold with the rolled Chlorosil was placed in an oven at $200^{\circ} \mathrm{C}$ for 12 hours to cure the Chlorosil. 
6. A thin piece of stiffer plastic with a mounting point for a cable was placed in a silicone pocket at the base of each lung.

7. The mold was removed from inside the Chlorosil-35 lungs through the breathing hole to which the trachea will be attached; this is possible because the material is highly flexible.

\section{II.B. Breathing Mechanism}

To modulate the volume, both a rubber "diaphragm" membrane stretched across the entire phantom base and a piston mechanism were considered. After preliminary testing, the diaphragm mechanism was found to be unreliable, so the piston mechanism was chosen. This consists of a large cylinder attached directly to the base plate of the phantom. A piston is driven back and forth in the cylinder using a linear actuator to create the volume change. The actuator works against the piston friction, water viscosity/inertia, and elastic forces in the lungs.

The mechanism consists of three major components: the piston and cylinder (figure 6), the base and mounting plates, and the linear actuator. Each is described below, before outlining their cumulative capabilities. The assembled mechanism is shown in figure 8 .
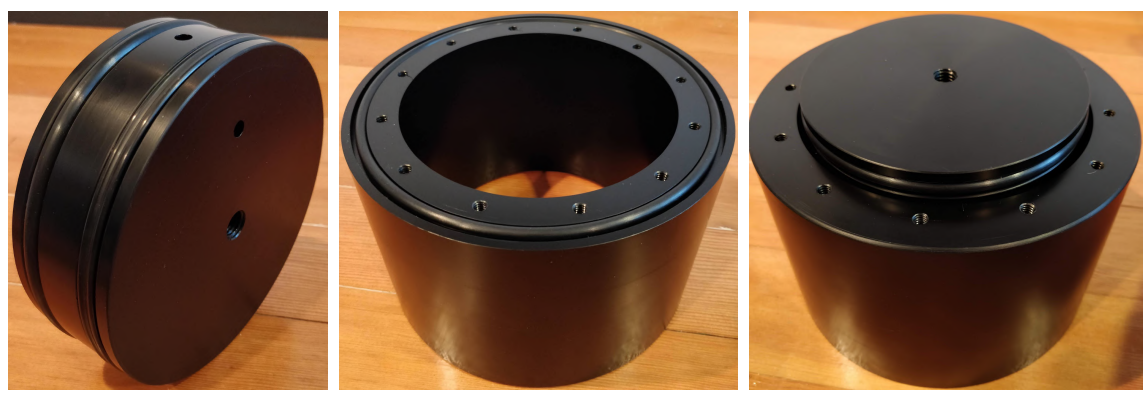

Figure 6: Left: Piston with dual O-rings; Center: Cylinder with O-ring to seal against base plate; Right: Piston in Cylinder. Threaded blind hole in piston to couple lung motion is visible.

\section{II.B.1. Piston and cylinder}

A key consideration in the piston and cylinder design was dimensional tolerance and stability. To ensure a good seal, specific dimensions with low tolerances had to be chosen, according to the Parker O-ring Handbook for dynamic sealing. To achieve and maintain the tight tolerances (eg. $\pm 0.1 \mathrm{~mm}$ over a $14 \mathrm{~cm}$ diameter) while avoiding attenuation and scatter from metallic components, both the piston and cylinder were manufactured from Delrin acetal 
plastic. Delrin is known to be dimensionally stable and machinable. It has a low water absorption of $0.25 \%$ (according to McMaster-Carr), which allows it to keep its shape when in contact with water. Furthermore, Delrin has a low friction coefficient of 0.2 , which reduces piston-cylinder friction and greatly decreases the required actuator load ${ }^{20}$. Beyond material choice, the cylinder was designed with thick, 27-mm walls to avoid any warping. A 3.8$\mathrm{mm}$ long chamfer at the mouth of the cylinder ensures easy insertion of the piston without scratching any of the surfaces important for the seal.

Two opposing factors played a role in determining the inner diameter of the cylinder. The larger the diameter, the smaller the piston displacement has to be for a given volume change, and thus the lower the speed requirement on the linear actuator. However, a larger piston is harder to machine within tolerance and to fit onto the base plate. A diameter of $14 \mathrm{~cm}$ was chosen as a compromise. At this diameter, the piston displacement for a given volume change is approximately equal to the axial lung extension for a given tidal volume ${ }^{7}$. For example, a piston movement of $3 \mathrm{~cm}$ leads to a volume change of $500 \mathrm{ml}$, which is a typical relaxed adult tidal volume. According to Wade et al. ${ }^{7}$, the lungs should very roughly extend on average $1.5 \mathrm{~cm}$ for $500 \mathrm{ml}$ tidal volume, though this varies widely between individuals. Ultimately, with the chosen dimensions, the change in the torso's (and thus also lungs') volume, $\Delta V$, is given by:

$$
\Delta V=\frac{\pi}{4} D^{2} \Delta x=\frac{\pi}{4}(14 c m)^{2}(\Delta x)
$$

where $D$ and $\Delta x$ are given in Fig. 2. The system's stroke is $8 \mathrm{~cm}$, so tidal volumes of up to $1232 \mathrm{ml}$ are possible, which constitute realistic deep breaths.

The piston has 2 O-ring grooves which ensure alignment inside the cylinder and provide a watertight dynamic seal (Fig. 7). The actuator-side groove is $6 \%(0.4 \mathrm{~mm})$ deeper, allowing for smaller \%-compression in the backup O-ring. This reduces the friction slightly but maintains sufficient level of seal to be used as backup. A vent hole connects the inter-O-ring space with the atmosphere in order to avoid pressure traps which can occur in double O-ring configurations. The chosen O-rings are size -429 , which have the largest cross-section for the diameter, and thus provide a large contact patch and effective sealing. To compromise between breakaway friction and running friction, 70A durometer Buna-N rubber O-rings were chosen. Buna-N rubber is stronger and more resilient and puncture resistant than other rubbers, making it a good choice for this wear-intensive application. In addition, it 


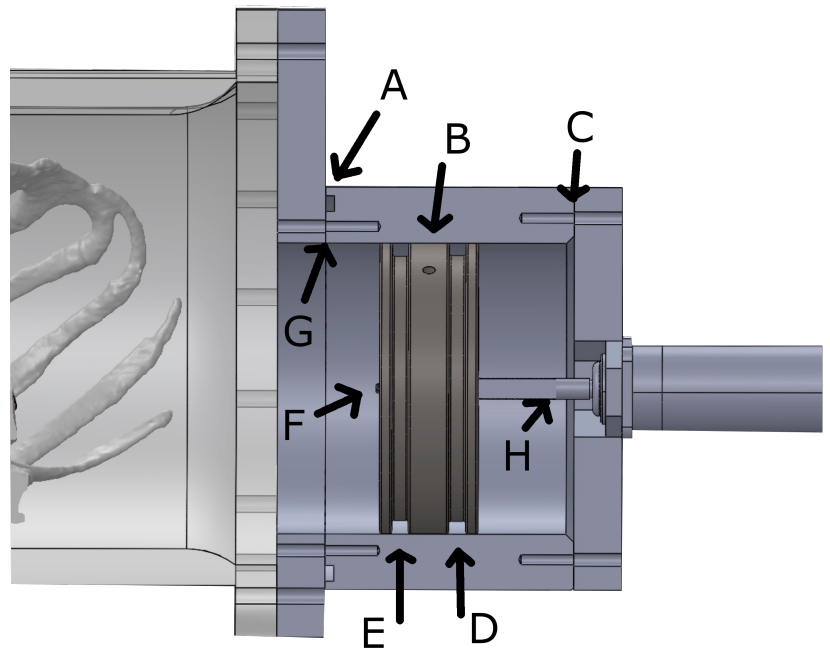

Figure 7: Cross-section of the piston drive mechanism. A: Cylinder face O-ring groove. B: Inter-O-ring vent hole. C: Actuator mounting plate screw holes. D: Backup O-ring groove. E: Main O-ring groove. F: Insert for mounting piston-lung motion-coupling cable. G: Cylinder to base plate mounting screw holes. H: Linear actuator threaded, male rod end mates directly with piston.

does not break down easily under influence of radiation. X- and circular-profile rings were tested, but no major difference in performance was detected.

The cylinder's inner surface was honed to 8-16 $\mu$ in RA roughness. An O-ring groove was milled into one end of the cylinder (part A in Fig. 7), with the mounting screws located radially inside the groove to avoid leaks through the screw holes. Twelve $1 / 4-20 \times 1$ " steel socket-cap screws fasten the cylinder to the base plate. The actuator mounting plate is attached in the same manner, but with no O-ring, and the flanged actuator is mounted directly to this plate. In this way, misalignment is minimized, and the whole structure is modular and easy to assemble and disassemble, for example to add or remove O-rings. The linear actuator has an $\mathrm{M} 12 \times 1.75$ threaded male rod end, which screws directly into the piston, leaving minimal clearance between piston and mounting plate in order to maximize the positional range of the piston. An identical threaded hole was made on the opposite side of the piston, into which an insert can be screwed to attach a cable to the lungs. Alternatively, a rod can be attached here to move an internal diaphragm, or the hole can be left empty. This gives flexibility in mounting and motion options. 


\section{II.B.2. Mounting Plates}

Two plates were made to mount the respiration mechanism to the existing Probe-IQ phantom, and to attach the linear actuator to the cylinder assembly. To ensure compatibility with Probe-IQ, a CT scan was taken of the phantom, and the base dimensions extracted as a SolidWorks file. The plate was fabricated from 1" thick polycarbonate to avoid backscatter that could be caused by metal. While the overall shape could be made using a waterjet cutter, a mill had to be used for all the screw holes since the waterjet piercing thick, relatively brittle polycarbonate causes local shattering. A large, central hole was included for the piston, as well as 36 1/4-20 holes for mounting the cylinder to the base plate and plate to the phantom base flange in a secure, sealed manner with the load distributed evenly. Finally, an 8-32 hole was drilled and tapped into the plate at the highest point of the torso cavity to purge air. The hole is sealed using a flat-head screw with a rubber gasket washer.

As the second plate is further away from the scanner's gantry, and supported fully by the cylinder, it was fabricated from 3mm-thick aluminum for weight, strength, and ease of fabrication. The plate was entirely waterjet cut, and included mounting points for the cylinder and actuator. The actuator has a front flange which is mounted directly to the plate to minimize the risk of misalignment. Furthermore, 4 additional holes were included to provide venting for the piston and avoid pressure build-up. Though no O-ring is present, this plate seals in any small leaks that potentially make it through the piston O-rings.

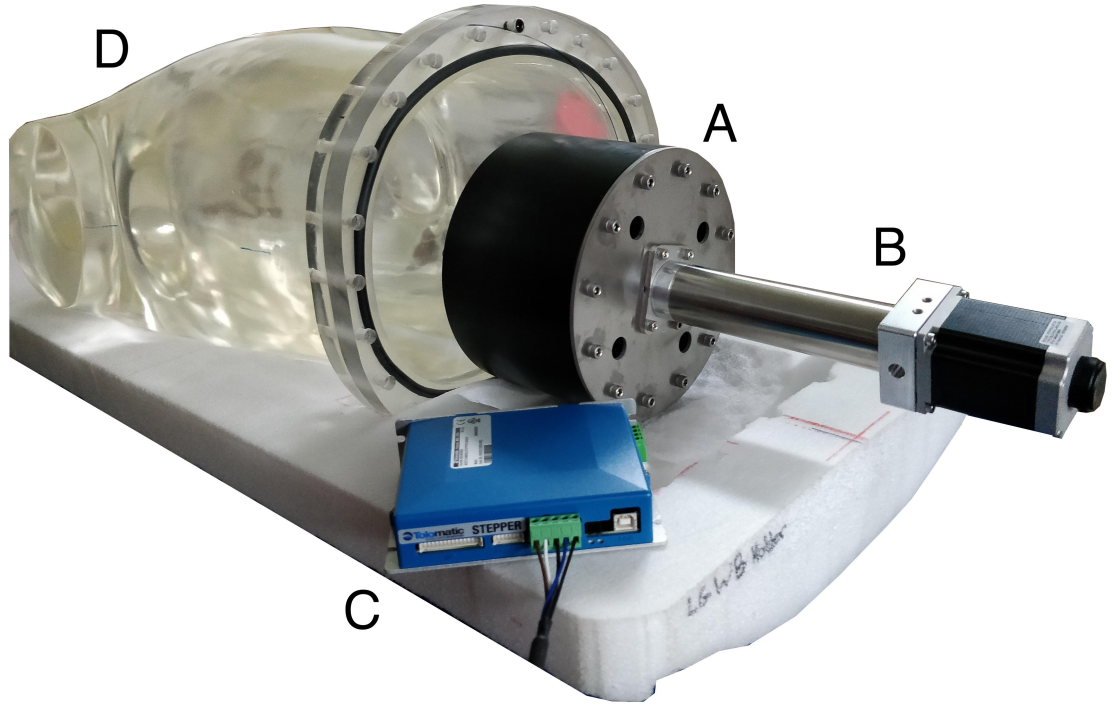

Figure 8: Assembled breathing mechanism (A. piston and cylinder, B. linear actuator, and C. stepper motor control box) with empty phantom (D). 


\section{II.B.3. Linear Actuator}

The actuator type and model were selected based on the criteria outlined in Table 2, as well as cost.

Table 2: Linear actuator design requirements

\begin{tabular}{|c|c|c|}
\hline Criterion & Desired & Chosen Actuator \\
\hline Speed & $54 \mathrm{~mm} / \mathrm{s}$ & $167 \mathrm{~mm} / \mathrm{s}$ \\
\hline Stroke & $90 \mathrm{~mm}$ & $90 \mathrm{~mm}$ \\
\hline Force & $900 \mathrm{~N}$ & $890 \mathrm{~N}$ \\
\hline Duty Cycle & $100 \%$ & $100 \%$ \\
\hline Durability & High & 5200 -scan estimated life \\
\hline Controllability & High & See in text \\
\hline Weight & Low & $\approx 1.5 \mathrm{~kg}$ \\
\hline
\end{tabular}

We set out to achieve a $1000 \mathrm{ml}$ tidal volume at 25 breaths per minute. While the piston was designed to provide up to $1232 \mathrm{ml}$ tidal volume, and the $90 \mathrm{~mm}$ actuator stroke supports the full range of piston motion, the actuator must be fast enough to reach 25 breaths per minute at this amplitude.

By using Equation 1 and noticing that a single breath involves both extension and retraction of the actuator, we find an expression for speed, $\dot{x}$, in $\mathrm{mm} / \mathrm{s}$ as a function of breathing rate, $\nu$, and amplitude, $V$, in breaths per minute and $\mathrm{ml}$ :

$$
\dot{x}=\frac{2 \nu V}{\frac{\pi}{4} 14^{2} \cdot 60} \approx \frac{\nu V}{461.8} \mathrm{~mm} / \mathrm{s}
$$

Hence, to fulfil the design objective, a linear speed of at least $54 \mathrm{~mm} / \mathrm{s}$ is required (assuming the actuator accelerates instantly, which is a reasonable assumption: the chosen actuator has a max acceleration of $\left.1667 \mathrm{~mm} / \mathrm{s}^{2}\right)$.

With some simplification, the required force is approximately the sum of the piston friction $\left(F_{f}\right)$, the water inertia $\left(F_{i}\right)$, and the elastic stretching of the lungs $\left(F_{e}\right)$ :

$$
F=F_{f}+F_{i}+F_{e}
$$

From the Parker O-Ring Handbook, $F_{f}=f_{c} L_{p}+f_{H} A_{p}$ where (assuming we are using the -429 O-ring):

- $f_{c}=0.933$ is the O-ring compression friction

- $L_{p}=17.29$ in $(43.92 \mathrm{~cm})$ is the seal rubbing surface length

- $f_{H}=10$ is the fluid pressure friction

- $A_{p}=3.97 \mathrm{in}^{2}\left(111.55 \mathrm{~cm}^{2}\right)$ is the projected seal area. 
Thus, the approximate piston friction is $F_{f}=250 \mathrm{~N}$.

To calculate the inertial force, assume the actuator accelerates to the $54 \mathrm{~mm} / \mathrm{s}$ velocity within $0.5 \mathrm{~cm}$. This corresponds to an acceleration of $a=\frac{(0.054 \mathrm{~m} / \mathrm{s})^{2}}{2 \cdot 0.005 \mathrm{~m}}=0.292 \mathrm{~m} / \mathrm{s}^{2}$. While most of the water in the phantom does not move much during the acceleration, at least the water that was in the piston will be accelerated, in addition to some water in the rest of the phantom. Suppose, then, that the total volume of water being accelerated is twice the maximum tidal volume - i.e. $2000 \mathrm{ml}$ - which is $2 \mathrm{~kg}$. Then

$$
F_{i}=(0.292 \mathrm{~m} / \mathrm{s})(2 \mathrm{~kg})=0.58 \mathrm{~N}
$$

This is negligible compared to the frictional force. Even if we took the whole $15 \mathrm{~kg}$ of water to be accelerating, it would still only constitute about $4 N$. This can therefore be ignored. Similarly, the static pressure on the piston can be ignored because the lungs in the water are at atmospheric pressure, and the piston sits at almost the same height as the lungs. Thus, the water pressure on the front of the piston and the air pressure on the back effectively cancel out.

The pressure required to stretch the lungs is more difficult to derive. To simplify the problem, let us assume that the lungs are cylindrical, and use the thin-wall approximation to find the hoop stress in one lung. This, however, also assumes that Hooke's Law holds for rubber-like elastomers. Merrit and Weinhaus ${ }^{21}$ found that this is not the case, and that after an initial linear section, the required pressure to increase the radius sharply falls off. Thus, Hooke's Law provides a rough, very conservative estimate. Let $\ell, r, t$ be the cylindrical lung's length, radius, and wall thickness respectively, and $\ell_{0}=200 \mathrm{~mm}, r_{0}=52 \mathrm{~mm}$, and $t_{0}=2 \mathrm{~mm}$ be the initial values. These give the expected initial volume of $1700 \mathrm{ml}$, as well as an initial material volume of $V_{0}^{\text {mat }} \approx 2 \pi r_{0} t_{0}\left(\ell_{0}+r_{0}\right)=21000 \mathrm{~mm}^{3}$. Using the incompressibility of rubber, $V_{0}^{\text {mat }}=V_{f}^{\text {mat }}$, so the wall thickness relates to the length and radius by:

$$
t \approx \frac{21000}{r(\ell+r)}
$$

Now using hoop stress, $\frac{r \Delta P}{t}=\sigma_{H}$, and Hooke's Law, $\sigma_{H}=\epsilon_{H} E=\frac{t_{0}-t}{t_{0}} E$, we obtain an expression for the required pressure differential applied by the piston:

$$
\Delta P=\frac{t\left(t_{0}-t\right)}{r t_{0}} E
$$

Combining Equations 5 and 6:

$$
\Delta P=\frac{21000}{r^{2}(\ell+r)}\left(1-\frac{21000}{r t_{0}(\ell+r)}\right) E
$$


This pressure is exerted evenly across the piston surface, so using $F=P A$ we find $F_{e}$ :

$$
F_{e}=(0.14)^{2} \frac{\pi}{4} \frac{21000}{r^{2}(\ell+r)}\left(1-\frac{21000}{r t_{0}(\ell+r)}\right) E
$$

The elastic modulus, $E$, of silicone rubbers can be estimated roughly from the Shore A durometer using Ruess' Equation: $\log _{10} E=0.0235 S-0.6403^{22}$. Since the lung silicone has Shore A hardness $35, E \approx 1.5 \mathrm{MPa}$. Thus we can finally calculate the force required to stretch the lungs. We want a $1000 \mathrm{ml}$ volume increase and about $6.6 \mathrm{~cm}$ lung extension ${ }^{7}$, so we take $\ell=266 \mathrm{~mm}$ and $r=56.8 \mathrm{~mm}$. Then $F_{e}=199 \mathrm{~N}$.

According to Equation 3, then, a conservative estimate for the maximum force required from the actuator is $F=450 \mathrm{~N}$. Applying a factor of safety of 2, considering that these calculations were approximate, piston friction could increase with wear, foam around the lungs could increase the pressure requirement, and a second O-ring could be added, with unknown effect, the linear actuator should be able to supply around $900 \mathrm{~N}$ of force.

To move the piston, a lead-screw actuation mechanism is preferred because of its superior controllability over pneumatic cylinders and other types of linear actuators. The phantom has to breathe continuously for the duration of the scan, which can be around 30 minutes, so the duty cycle has to be $100 \%$ for extended periods of time. Many actuators use ACME screws, a specific thread geometry which can only operate at about $15 \%$ duty cycle. Instead, a ball screw mechanism, which has much less friction, allows for continuous use and a long lifetime.

Based on these criteria, an ERD15 (Tolomatic, USA) ball-screw linear actuator was chosen, along with a Nema-23, 2-stack stepper motor with encoder feedback. This relatively low-cost actuator is designed to run continuously and fulfills the load, stroke, and speed requirements. Assuming we carried out 30 minute scans at the calculated force, at 25 breaths per minute and $1000 \mathrm{ml}$ tidal volume, the actuator would have an estimated life of 5200 scans (according to manufacturer specifications ${ }^{23}$ ). The ERD's low profile and simplicity allow it to be mounted as seen in Fig. 8 .

According to the manufacturer specifications, the actuator position is accurate to within $0.03 \mathrm{~mm}$ per $\mathrm{cm}$ of movement ${ }^{23}$, which translates to a volumetric error of up to $0.3 \mathrm{ml}$ per $100 \mathrm{ml}$ volume change, using Equation 1. In addition, the 1.8\% step stepper motor combined with the 5mm-lead ball screw mechanism, as well as 2000-count encoder feedback facilitate exact velocity and acceleration control. 
The force analysis was verified during testing by running the actuator at $40 \%$ force, where it stalled, and then at $60 \%$, where it ran smoothly. This implies the required force is around $50 \%$ of the actuator's force capability, which, given the chosen safety factor of 2 , means the required force corresponds closely to the derived value.

\section{II.C. Software and Electrical Design}

The Tolomatic ERD15 actuator is driven by a stepper motor with encoder feedback, which is in turn powered by a $120 \mathrm{~W}, 48 \mathrm{~V}$ power supply, and controlled by stepper driver hardware from Tolomatic. The goal in this control system is to create realistic, consistent, repeatable lung motion with flexibility in breathing rate and amplitude, as well as the ability to include breathing inconsistencies for added realism. Two distinct control methods were created for the phantom and are described below. The first is simple and fast to set up while the second provides more realism, so each is useful in different applications.

First, a free Windows application (described below) can be used to communicate with the stepper driver over USB to configure the actuator. This option is explored in-depth below. The second option is analog position control. Here, a $0-10 \mathrm{~V}$ analog signal can be input to the driver, which moves to the corresponding position. Control through ethernet and Modbus RTU over RS-485 are also possible, but neither was investigated in this work.

\section{II.C.1. USB Interface}

The actuator manufacturer provides a software interface called Tolomatic Motion Interface (TMI) to communicate with its stepper controllers over USB. While the software is primarily used to configure the driver by setting acceleration, deceleration, maximum velocity, error states and more, it can also be used to directly control the actuator's movement. In 'Indexing Mode' up to 16 moves can be defined, each with a certain acceleration, deceleration, velocity, and goal position. Any of these moves can then be chosen and combined in any order to form a repeating series of up to 16 moves. Taking the simplest example, one may define just 2 moves, one to $45 \mathrm{~mm}$, and one to $1 \mathrm{~mm}$, and cycle between these two positions indefinitely. By combining more positions, however, and altering their accelerations and decelerations, a wide variety of breathing patterns can be achieved, including realistic respiratory cycles.

The problem with this method is that visualizing what the respiratory cycle will look like based on a series of linear position, velocity, and acceleration values is difficult. A graphical user interface (GUI) was therefore developed in Python to allow motion planning 
and visualization of the lung volume as a function of time (figure 9).

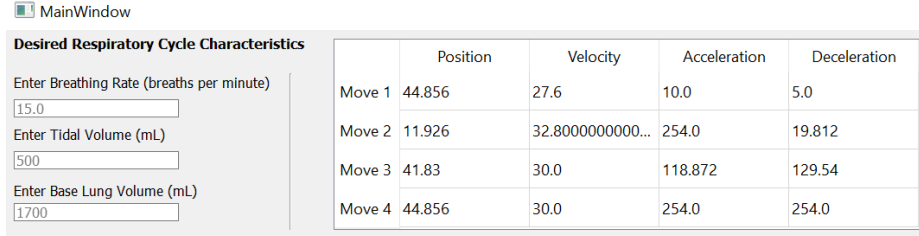

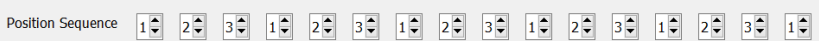
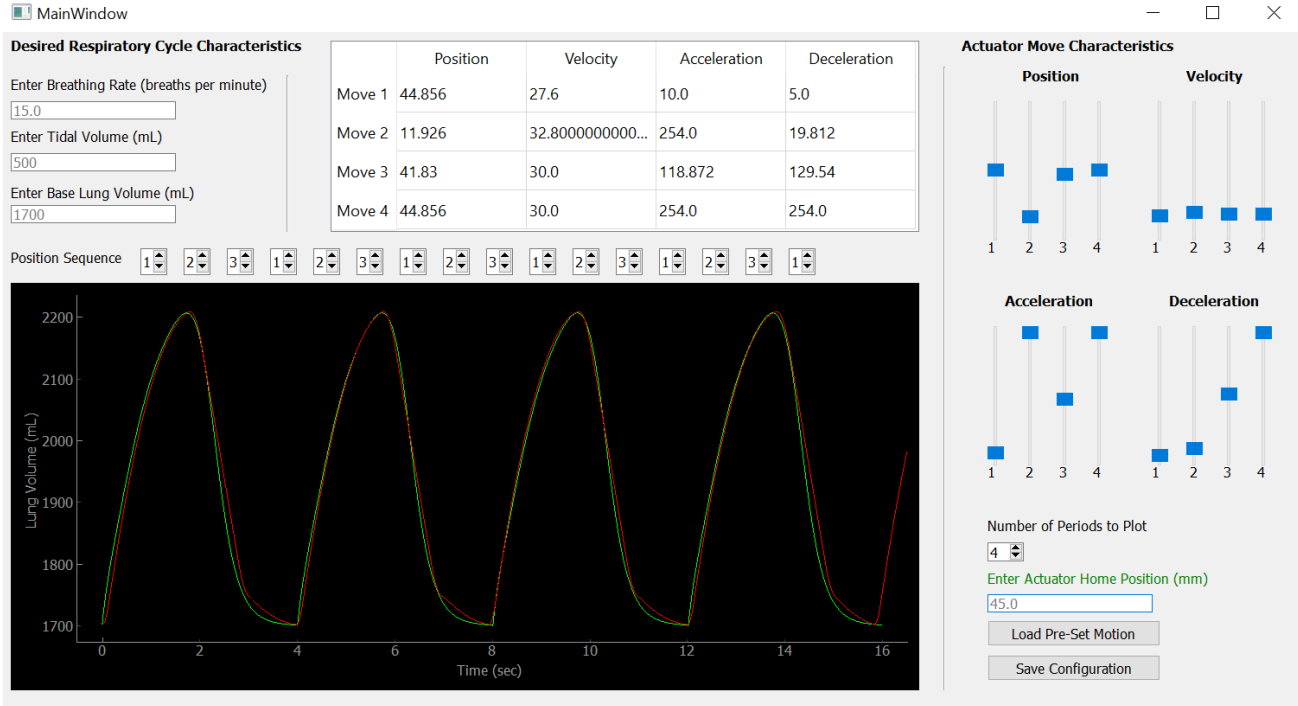

Figure 9: Screenshot of motion planning application. The green curve is the desired respiratory cycle, while the red is the pattern carried out by the phantom based on the GUI inputs.

In the application, the user can define the breathing rate, amplitude, and base lung volume. We used published models ${ }^{24}$ to identify a physiologically representative equation for a respiratory curve (Equation $9, x=$ amplitude, $t=$ time), which can be scaled and shifted to match the desired, user-defined breathing parameters. This curve is plotted in green in figure 9 .

$$
x=e^{-1.71668 t} \frac{0.0106+2.3313 t+0.535 t^{2}+3.9594 t^{3}}{2.3062+0.3517 t-0.6487 t^{2}+0.1177 t^{3}}
$$

The user can then define up to 4 moves with position, velocity, acceleration, and deceleration, and place them in any order. The corresponding motion profile is generated through iterative kinematic simulation of the actuator, and plotted in real time, in red in Fig. 10, as the parameters are changed. With the red actuator plot overlaid onto the green desired plot, the optimal set of motion parameters can be determined. It is also possible to save configurations in automatically-formatted JSON files to be loaded later.

This control method is simple because the respiratory cycle is visualized, input into user-friendly software, and relayed to the actuator directly over USB. While a variety of motions can be achieved in this manner, it is nonetheless limited by the discrete nature of a set of individual moves. To overcome this problem, at the cost of simplicity, analog control can be used. 


\section{II.C.2. Analog Position Control}

The second method controls the ERD15 actuator using a 0-10V analog signal. The voltage range is mapped onto the positional range of the actuator so that a given voltage causes the actuator to move to that position. The move occurs with predefined acceleration, deceleration, and maximum velocity, which can be configured using the Tolomatic Motion Interface software, but is non-blocking; i.e. a new position command can be given before the current position has been reached, and the actuator will immediately move towards the new position. In this way, arbitrary continuous waveforms can be realized by the actuator, if they do not exceed the configured acceleration and velocity values.

Figure 10 shows the setup used to generate the analog signal. The voltage range is configurable using the Tolomatic software, and was set to $0-3.3 \mathrm{~V}$, which was provided by an MCP4725 Digital to Analog Converter (DAC), controlled via the I2C GPIO pins of a Raspberry Pi microcomputer. The encoder feedback is read by the Raspberry Pi, and any control software is run on the Raspberry Pi.

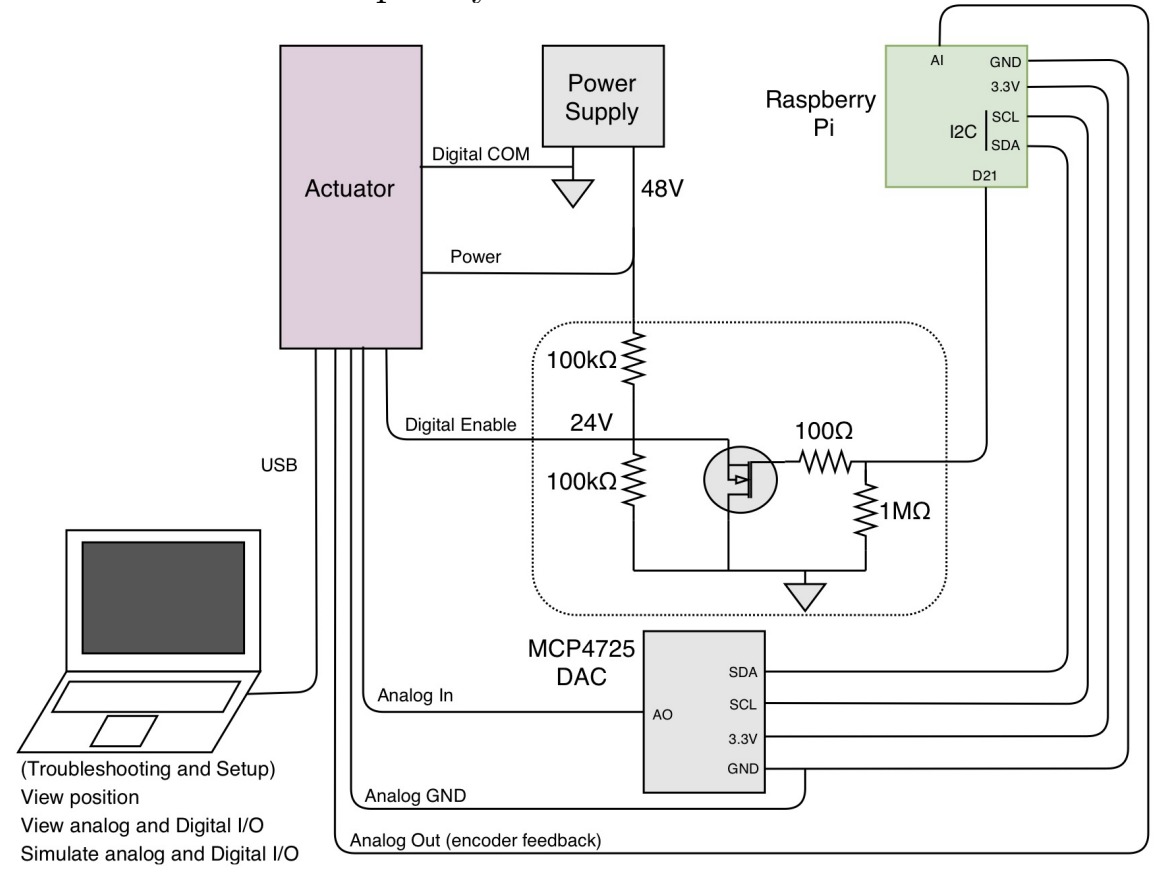

Figure 10: Schematic of analog position control setup.

For the actuator to respond to the analog input voltage, analog control must be enabled through a digital input. The digital I/O operates on $28 \mathrm{~V}$ logic (18-28V is considered high) with respect to the digital common pin $(\mathrm{COM})$. Thus, digital $\mathrm{COM}$ is tied to the power supply ground, and a voltage divider is used to provide the $24 \mathrm{~V}$ needed to enable motion. 
An N-channel MOSFET whose gate voltage is set by the Raspberry Pi switches the enable input between 0 and $24 \mathrm{~V}$. The MOSFET gate is pulled down to ground through a $1 \mathrm{M} \Omega$ resistor and attached to the $\mathrm{Pi}$ through a $100 \Omega$ resistor to prevent parasitic ringing. The voltage divider uses $100 \mathrm{k} \Omega$ resistors so the maximum current draw is $0.24 \mathrm{~mA}$, which does not affect the operation of the actuator.

With this setup, arbitrary waveforms can be saved as CSV files on the Raspberry Pi. A Python program converts the volumetric data to actuator positional curves using the piston geometry, and then to voltages using the voltage-position mapping. The voltages are sent via $\mathrm{I} 2 \mathrm{C}$ to the $\mathrm{DAC}$, where they are converted to analog voltages and input into the actuator, thus generating the desired lung motion.

\section{Results: Testing and Validation}

To validate the efficacy of the piston and lung design, testing of the complete assembly was performed. The primary criteria for evaluation were realism in the trajectory, symmetry between the lungs, consistency of movement, and controllability, as well as validation of the force requirements and mechanical integrity.

The phantom was set up in the single O-ring configuration with the lungs not attached to the piston, and was then filled with water. Filling was straightforward using the neck ports. The lungs maintained their position and shape when opened to the atmosphere, and the piston was then run back and forth continuously for 30 minutes with cameras mounted above and on either side of the phantom. No water leakage to outside of the phantom was observed. The piston was first set to move at $30 \mathrm{~mm} / \mathrm{s}$ and then $40 \mathrm{~mm} / \mathrm{s}$, both with a $58 \mathrm{~mm}$ amplitude. This corresponds to $892 \mathrm{ml}$ tidal volume at 15 and 21 breaths per minute, respectively.

The resultant trajectory is shown in figure 11. The videos are available in the open source materials. Qualitatively, the breathing looked realistic, and included some rib motion and flexing, which adds to the realism. The lungs' maximum and minimum positions were ascertained from the videos at all angles. There is little difference between the right and left lung, as seen when viewed from above (figure 11, right image).

Analysis of the actual displacements allows comparison to the average trajectories previously determined for adult males. The results are given in Table 3, in comparison with previously published values ${ }^{7}$. These results show that the lungs naturally expand slightly

III. RESULTS: TESTING AND VALIDATION 


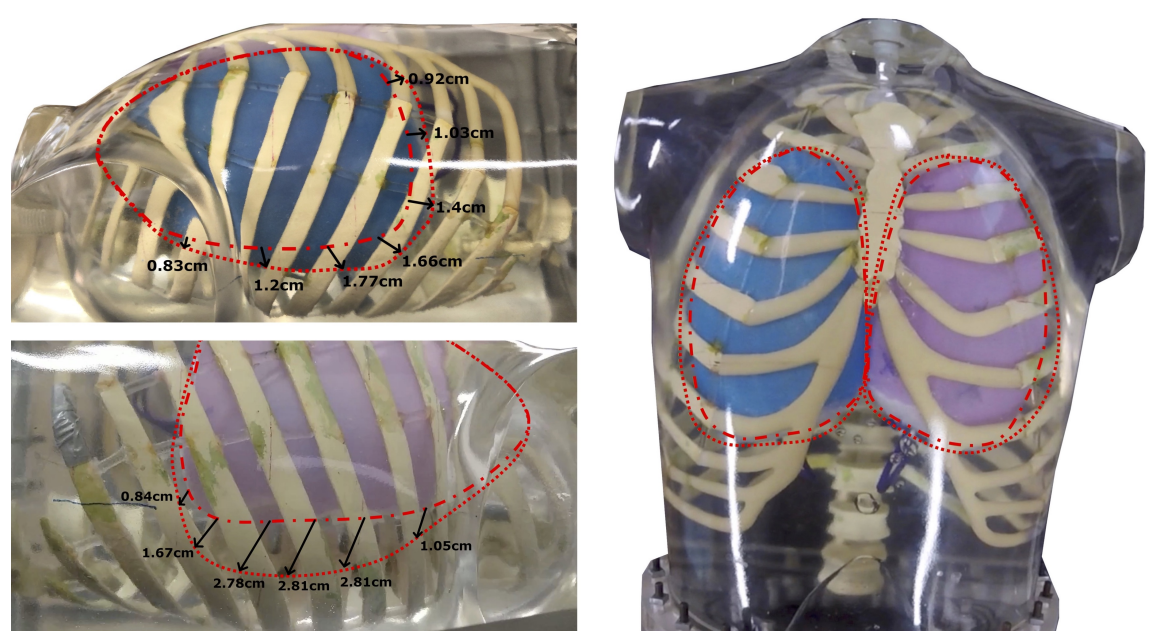

Figure 11: Screen grabs from the breathing test. The dotted lines indicate maximum inhalation, while the dashed lines are maximum exhalation. The tidal volume is $892 \mathrm{ml}$. Note that the camera angle on the right and left lungs are slightly different to show a different cross-section of the trajectory. The view of both lungs show that they move almost identically.

more in circumference and extend less axially than desired. However, they are within the reasonable realistic adult male range given by Wade's measurements and his observation of variability between individuals.

Table 3: Lung Trajectory Comparison. The 'scaled' column is the measured parameters normalized to match Wade's tidal volume for ease of comparison (assuming the parameters scale approximately linearly with volume in this range)

\begin{tabular}{|c|c|c|c|}
\hline Parameter & ${\text { Wade et } \text { al. }^{\mathbf{7}}}$ & Phantom & Scaled \\
\hline Tidal Volume & $799 \pm 210 \mathrm{ml}$ & $892 \mathrm{ml}$ & $799 \mathrm{ml}$ \\
\hline Axial Extension & $1.7 \pm 0.26 \mathrm{~cm}$ & $1.4 \mathrm{~cm}$ & 1.3 \\
\hline Circumference Increase & $6.8 \pm 2.2 \mathrm{~cm}$ & $8.4 \mathrm{~cm}$ & $7.5 \mathrm{~cm}$ \\
\hline
\end{tabular}

Finally, the consistency of the motion was analyzed by tracking three markers placed on the left lung for 20 breaths using MATLAB. The frames were binned using the known frame rate of the video and the set breathing rate such that only frames showing the lungs at $100 \%, 75 \%, 50 \%, 25 \%$, and $0 \%$ inhalation were used. The motion of each point formed a scatter plot through which a line was fitted, and the root-mean-squared (RMS) error was calculated as an indicator of the motion's consistency. The resulting RMS errors were $0.74 \mathrm{~mm}, 0.52 \mathrm{~mm}$, and $0.54 \mathrm{~mm}$ from the straight line. Note that the tracking error in the position measurements was about $\pm 1 \mathrm{~mm}$, so the results show that the lung motion was consistent between the breaths. 
After one of the tests that included a total of 40 minutes of almost continuous motion, the phantom was emptied, and the drive mechanism was dismantled and inspected. There were no signs of wear in the mechanism, which would have indicated a problem. The Oring remained very well lubricated, and the cylinder wall behind the O-ring was dry. It is expected that with continued good lubrication and occasional O-ring replacement, the chance of leakage will remain very low. Since the phantom rests on an absorbent sheet throughout operation and is left in isolation until the isotopes have decayed to background levels, individual drops that may get through the piston do not represent a concern, especially as they remain contained within the cylinder. However, the second O-ring can also be installed on the piston to further improve the seal.

\section{Discussion}

We have developed and validated the main aspects of the phantom design and usability, including materials, fabrication, couplings, actuation, dynamics, control, and procedures such as filling the phantom. These elements have been shown to work together well to achieve the desired purpose of modeling non-rigid lung motion inside an anthropomorphic phantom. The design objectives of consistent, controllable, symmetric, and realistic motion have been met, though there are a few aspects that can be improved in future work.

In normal respiration, lungs typically expand more axially than in cross-section ${ }^{7}$. In the developed phantom, the lung expansion was more uniform. Thus, artificial lesions attached to inferior lobes of the manufactured lungs will travel a shorter path on average compared to reality (for a similarly-sized person). Two approaches can be taken to increase the axial expansion. First, the lungs could be coupled directly to the piston using an elastic cord. Making this coupling looser or tighter would produce more or less axial extension, while also keeping the lungs centered in the antero-posterior direction. Alternatively, during the lung fabrication the wall material (Chlorosil) can be strategically rolled thinner in parts that should expand more.

In addition, though the ribs flex to accommodate the lung expansion, the torso shell is rigid, so the lungs are forced to expand dorsally instead of the chest rising as it does in humans. However, by attaching the lungs to the spine so they do not float up when the phantom is placed in a supine position, they can expand upwards during inhalation. In this way, lesions placed on the lungs near the chest retain the expected, anatomically correct

\section{DISCUSSION}


trajectory.

A limitation of the phantom is that the lungs are filled with air, which makes it impossible to place artificial lesions inside the lungs. Furthermore, the $511 \mathrm{keV}$ gamma attenuation coefficient is close to 0 inside the lungs, whereas in reality, it should be around $40 \%$ that of soft tissue $^{17}$. We believe that both limitations can be addressed by filling the lungs with light and high-springback foam. If the foam is added inside the lungs in the compressed state, it can hold artificial lesions in place and expand with together with lungs, while also increasing the attenuation coefficient. The same method can be used to fill the space outside the lungs to introduce realistic image heterogeneity through small air bubbles caught in the foam and to facilitate organ placement (liver and heart), as shown previously in the Probe-IQ. The foam can also transmit the lung motion to the other organs, leading to liver motion. Here the foam's effect on lung expansion, and the potential for all the bubbles to escape the foam under the constant motion must be assessed.

The described phantom design, materials and principle of operation can facilitate the construction of similar phantoms elsewhere, and guide the development of a next iteration of phantoms for PET/CT imaging. Once COVID-19 restrictions at our center are eased, CT scans will be performed at different phases of the respiratory cycles to more precisely characterize the motion. Pilot PET scans will also be performed to characterize the effect of lung motion on lesion quantification in a realistic environment. In future work, we will report the results of initial CT and PET scans, and investigate methods to further improve the realism of lung motion.

\section{Conclusion}

In this work we presented a method and mechanical design to incorporate anatomicallyaccurate, elastic lungs and respiratory motion into an anthropomorphic phantom for PET imaging. The proposed physical principle of operation, materials, couplings and electronics have been validated. A new method to fabricate elastic lung models using 3D-printed molds was established and tested, and a software interface to plan and visualize these breathing patterns was developed, with two separate control schemes. The design can achieve a variety of respiratory wave-forms with up to 25 breaths per minute at $1232 \mathrm{ml}$ tidal volume, and is expected to contribute to studies evaluating the effect of respiratory motion on detectability and quantification of cancer lesions. 


\section{Open Source}

To promote further development of PET and phantom technology, we have opensourced the entire design, including all CAD files, code, engineering drawings, and more, with supporting documentation. This material can be found here: https://github.com/dgblack/robotPhantom.

\section{Acknowledgements}

We gratefully acknowledge support by Barber Prosthetics (Vancouver, Canada) in all aspects related to lung manufacturing. The linear actuator for initial testing was provided by Robert de Rot at PQ Systems Ltd (Vancouver, Canada). We also gratefully acknowledge the Engineering Physics Project Lab at the University of British Columbia for financial and expertise support, as well as the BC Cancer Foundation for financial support.

\section{References}

1 P. E. Valk, E. Abella-Columna, M. K. Haseman, et al., Whole-body PET imaging with [18F]fluorodeoxyglucose in management of recurrent colorectal cancer., Arch. Surg. 134, 503-11; discussion 511-3 (1999).

2 S. A. Nehmeh, Y. E. Erdi, C. C. Ling, et al., Effect of respiratory gating on reducing lung motion artifacts in PET imaging of lung cancer, Med. Phys. 29, 366 (2002).

3 L. Guerra, E. De Ponti, F. Elisei, et al., Respiratory gated PET/CT in a European multicentre retrospective study: added diagnostic value in detection and characterization of lung lesions, European Journal of Nuclear Medicine and Molecular Imaging 39, 1381-1390 (2012).

4 R. Manber, K. Thielemans, B. Hutton, et al., Clinical impact of respiratory motion correction in simultaneous PET/MR, using a joint PET/MR predictive motion model, Journal of Nuclear Medicine 59, 1467-1493 (2019).

5 G. El Fakhri, Y. Rakvongthai, Magnetic resonance-based motion correction for quantitative PET in simultaneous PET-MR imaging, PET Clinics 12, 321-327 (2017).

6 M. Dawood, N. Lang, X. Jiang, and K. P. Schäfers, Lung motion correction on respiratory gated 3-D PET/CT images., IEEE Trans. Med. Imaging 25, 476-85 (2006). 
7 O. Wade, Movements of the thoracic cage and diaphragm in respiration, Journal of Physiology 124, 193-212 (1954).

8 F. Nayyeri, A review on motion correction methods in PET/CT images for detection of cancer cells, Acta Medica Bulgarica 42, 68-78 (2015).

9 R. Schmitz, A. Alessio, and P. Kinahan, The physics of PET/CT scanners, Technical report, University of Washington.

10 A. Chi and N. Nguyen, 4D PET/CT as a strategy to reduce respiratory motion artifacts in FDG-PET/CT, Frontiers in Oncology 4, 205 (2014).

11 S. S. Korreman, Motion in radiotherapy: photon therapy, Phys. Med. Biol. 57, R161R191 (2012).

12 X. Zhang, Z. Xie, E. Berg, et al., Total-Body Dynamic Reconstruction and Parametric Imaging on the uEXPLORER, J. Nucl. Med. 61, 285-291 (2020).

13 R. J. Gillies, P. E. Kinahan, and H. Hricak, Radiomics: Images Are More than Pictures, They Are Data, Radiology 278, 563-577 (2016).

14 K. Bolwin, B. Czekalla, L. Frohwein, F. Büther, and K. Schäfers, Anthropomorphic thorax phantom for cardio-respiratory motion simulation in tomographic imaging, Physics in Medicine and Biology 63 (2018).

15 I. Chrysanthou-Baustert, I. Polycarpou, O. Demetriadou, et al., Characterization of attenuation and respiratory motion artifacts and their influence on SPECT MP image evaluation using a dynamic phantom assembly with variable cardiac defects, Journal of Nuclear Cardiology 24, 698-707 (2017).

16 D. Kadrmas, M. Casey, M. Conti, B. Jakoby, C. Lois, and D. Townsend, Impact of timeof-flight on PET tumor detection, Journal of Nuclear Medicine 50, 1315-23 (2009).

17 D. Kadrmas, M. Casey, N. Black, J. Hamill, V. Panin, and M. Conti, Experimental comparison of lesion detectability for four fully-3D PET reconstruction schemes, IEEE Trans Med Imaging 28, 523-34 (2009). 
18 National Institute of Standards and Technology, X-Ray mass attenuation coefficients. https://physics.nist.gov/PhysRefData/XrayMassCoef/tab4.html

19 Wikipedia, Lung volumes. https://en.wikipedia.org/wiki/Lung_volumes D. Bhatt and K. Mistry, Experimental study of friction under different variables on a piston-cylinder assembly. D. Merrit and F. Weinhaus, The pressure curve for a rubber balloon, American Journal of Physics 46, 976-977 (1978). K. Larson, Can you estimate modulus from durometer hardness for silicones?, 2019. Tolomatic, ERD Electric Cylinder Features and Options. www.tolomatic.com and exponential flow waveforms, Anaesthesia 52, 1182-1194 (1997). 